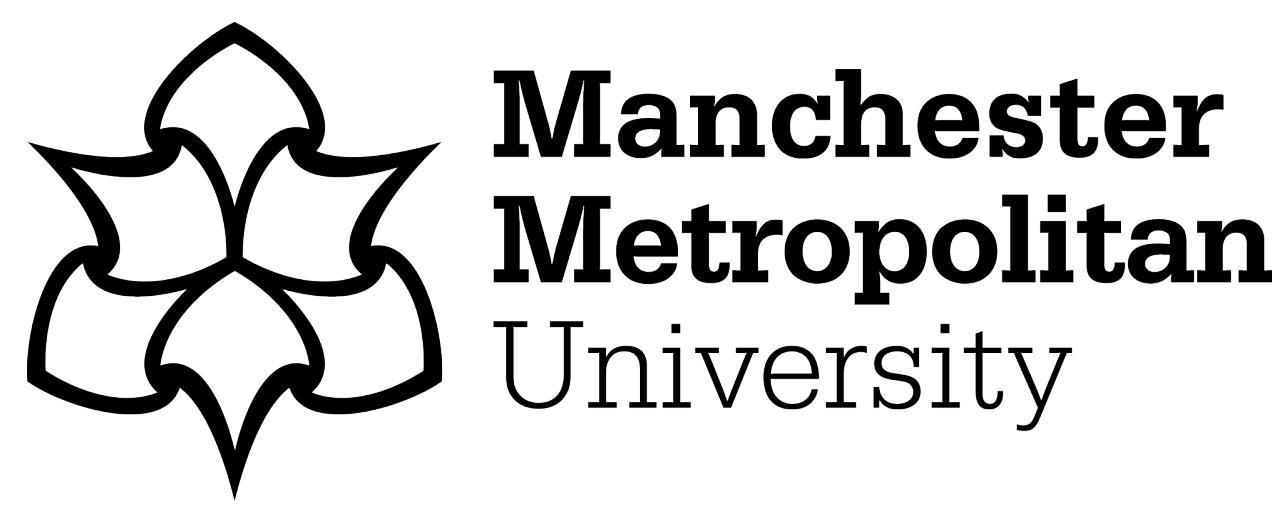

Sánchez, María B, Loram, lan, Holmes, Paul, Darby, John and Butler, B Penelope (2018) Quantification of head motion in children with cerebral palsy when testing segmental trunk control. In: 2018 IX International Seminar of Biomedical Engineering (SIB), 16 May 2018 - 18 May 2018, Bogota, Colombia.

Downloaded from: https://e-space.mmu.ac.uk/622645/

Version: Accepted Version

Publisher: IEEE

DOI: https://doi.org/10.1109/sib.2018.8467741

Please cite the published version 


\title{
Quantification of head motion in children with cerebral palsy when testing segmental trunk control
}

\author{
Sánchez, María B; Loram, Ian; Holmes, Paul; Darby John; Butler, Penelope B. \\ Manchester Metropolitan University \\ Manchester, UK \\ m.sanchez.puccini@mmu.ac.uk
}

\begin{abstract}
The development of objective assessment tools to complement subjective evaluations could have a major impact in the evaluation of the consequences of neuromotor disabilities, specifically in the assessment of trunk control in children with cerebral palsy (CP). Current assessments are subjective and typically assess the general control status of a child under unbalancing situations. Only the Segmental Assessment of Trunk Control (SATCo) evaluates static, active and reactive control at six discrete trunk segmental levels and free sitting to identify the topmost trunk segment where the child does not demonstrate control. The characteristics of the SATCo make it an ideal start point to generate an objective measurement tool of control. Motion of the head is one of the main characteristics observed during a SATCo to determine the child's segmental level of control. This pilot study measured head motion during a SATCo to determine if the measurement reflected the clinical observations. Three children with different degrees of neuromotor disability were tested using the SATCo while a video camera recorded sagittal plane movements. A Head segment was defined by markers on the ear tragus and temporal fossa in vertical line with the ear when the head was aligned. Head segmental angles were calculated in relation to a real vertical, and the cumulative displacement was normalised by time (deg-s-1). One segment where external support was provided high on the trunk (Upper-Thoracic, UT) and one where external support was at the pelvis i.e. low on the trunk (Lower-Lumbar, LL) were selected for the analysis. Overall, head motion values were larger for the lower trunk tests (LL) than for the tests at the higher segment (UT) with greater external support provided. The poorer lumbar control of Child 2 and Child 3 was demonstrated in both active $\left(39.9^{\circ} \cdot \mathrm{s}-1,23.3^{\circ} \cdot \mathrm{s}-1\right)$ and reactive tests $\left(37.3^{\circ} \cdot \mathrm{s}-1,13.8^{\circ} \cdot \mathrm{s}-1\right)$. The results also showed how increasing task complexity (static to reactive) and reducing the level of support (UT to LL), increased head motion in the presence of a neuromotor disability. The results presented here corroborated the clinical evaluation of the SATCo. Head motion will have small values when children with $C P$ are tested at a trunk segmental level where control has already been learnt or where external support is provided; children with poorer lumbar control will have an increased head motion when testing is below their trunk segmental level of assured control. This video-based approach represents a step towards the development of an objective tool for the assessment of head/trunk control in children with $\mathrm{CP}$.
\end{abstract}

Keywords-Objective assessment; trunk control; cerebral palsy; head motion; video-recording

\section{INTRODUCTION}

The development of objective, clinically friendly assessment tools has an important role in the validation of physiotherapeutic interventions. Currently, the outcomes resulting from an intervention are generated by clinical assessments that are almost exclusively based on subjective criteria; these assessments are validated but nevertheless rely upon the assessor's expertise and consistency. The development of objective assessment tools to complement subjective evaluations in physiotherapy, as in other medical and complementary fields, is of paramount importance in the requirement for evidencebased practice (EBP). EBP integrates the best research evidence, individual clinical expertise and patient choice in making decisions about the care of patients [1]. EBP is desirable to help standardise intervention protocols and can provide validated support to decision making.

Objective measurement tools could thus have a major positive impact in many different areas of physiotherapy such as in the assessment of patients with neuromotor disabilities. Neuromotor disabilities, such as cerebral palsy (CP), compromise a child's movement ability, as a consequence of the neurological damage [2]; this motor compromise results in functional limitations, especially where there is head and trunk involvement. Assured trunk control is mandatory for effective function in typically developing children and in those with neuromotor disability [3]; the functional limitations that derive from poor trunk control are likely to adversely impact a child's performance of activities of daily living and/or their participation in social activities [4].

Specific assessments of how a child's head and trunk control and sitting ability are affected as a consequence of the motor involvement are essential to the development of an appropriate and comprehensive therapeutic plan to enhance overall function. Head/trunk control of children with CP is most usually assessed in a physiotherapy practice through comparison with typically developing children and inferring control status from functional activities [5, 6]. Assessments typically test the child's control under unbalancing situations in sitting [7, 8], without consideration of the adjustments and compensations the child makes to maintain a balanced position (for example arching the back or supporting him/herself with the hands). Furthermore, most of these assessments consider the trunk as a single unit, 
which lacks important detail of the head/trunk control status of a child. In contrast, the Segmental Assessment of Trunk Control (SATCo) assesses control of the neutral vertical posture at six discrete trunk segmental levels (Head; Upper-Thoracic, UT; Mid-Thoracic, MT; Lower-Thoracic, LT; Upper-Lumbar, UL; Lower-Lumbar, LL) and Free Sitting [9]. Additionally, the SATCo includes static, active and reactive control giving a more complete and detailed picture of trunk control status; static control represents the ability of a person to maintain an independent sitting posture, active or anticipatory control looks at the response to internally generated movements while reactive control examines the response to externally generated perturbations (a nudge) and the restoration of a neutral upright sitting posture.

The SATCo is conducted in sitting with external support provided manually directly beneath the tested head/trunk segment. At each trunk segmental level, a subjective clinical evaluation is made of the demonstration or not of control of the neutral vertical posture above the support. Because of its characteristics, the SATCo represents an ideal starting point for the development of a clinically friendly video-based objective measurement of control status. In a clinical context, the movement of the head is one of the main characteristics observed to determine the child's trunk control status. This pilot study measured head motion during a SATCo to determine if measurement reflected the clinical observations of i) head motion with external support high on the trunk will result in small head motion values since control is largely externally provided and ii) head motion with external support low on the trunk will result in larger head motion values that are dependent on the child's trunk control status. If measurement of head motion corroborates the clinical observation, this may form an initial step towards the generation of an objective quantification of a subjective assessment.

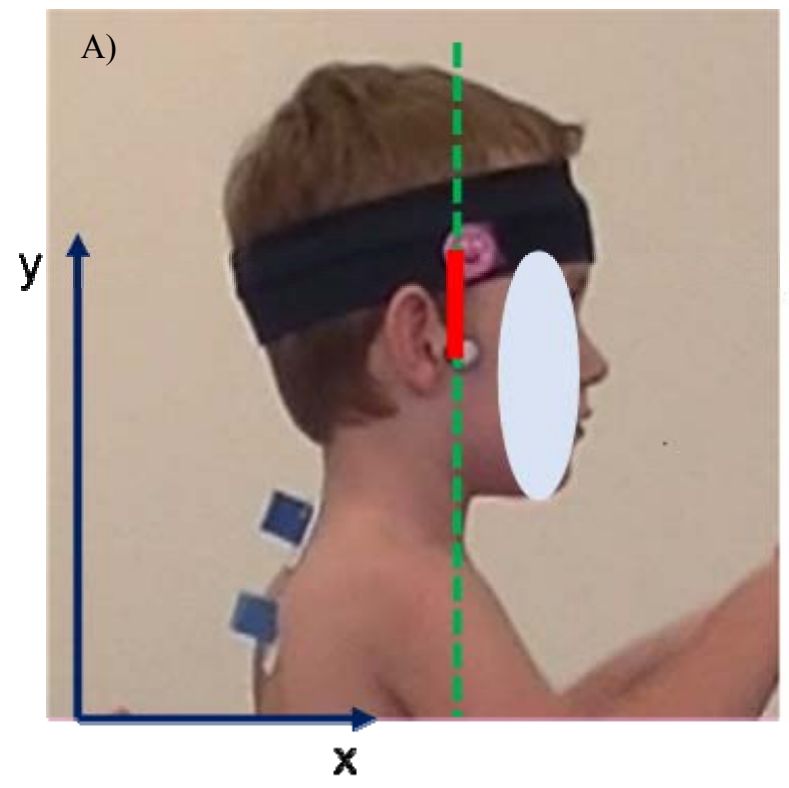

\section{MEthodS}

\section{A. Ethics}

Ethical approval for the study was obtained from the NHS Health Research Authority (NRES Committee South Central, United Kingdom) and from the Manchester Metropolitan University Ethics Committee. The study was conducted in accordance with the Declaration of Helsinki guidelines.

\section{B. Participants}

Three children with differing degrees of neuromotor disability were tested using the SATCo. Child 1 (4 years 1 month) was learning static, active and reactive control of the full trunk (i.e. sitting with no external support). Child 2 (4 years 5 months) and Child 3 (6 years 1 month) were both learning static, active and reactive control at the Upper-Lumbar segment (i.e. support at waist level).

\section{Apparatus and Measurements}

A video camera (JVC, HD Everio RX110) recorded sagittal plane movements at $25 \mathrm{~Hz}$. Markers were placed on the ear tragus and temporal fossa in vertical line with the ear when the head was aligned. These were used to define a head segment (Fig. 1A). For each test, the coordinates of the markers were tracked using a specialised software (Dartfish 7, TeamPro 7.0). These coordinates were used to calculate the head segmental angle in relation to a real vertical (Fig. 1). Cumulative absolute displacement from the vertical normalised by time $(\mathrm{deg} \cdot \mathrm{s}-1)$ was calculated for each trial. The Upper-Thoracic (UT) and LowerLumbar (LL) segmental levels were selected for comparison as they represented i) where all three children had clinically demonstrated control (UT), and ii) where control had been clinically demonstrated in Child 1 but not in Child 2 or Child 3.

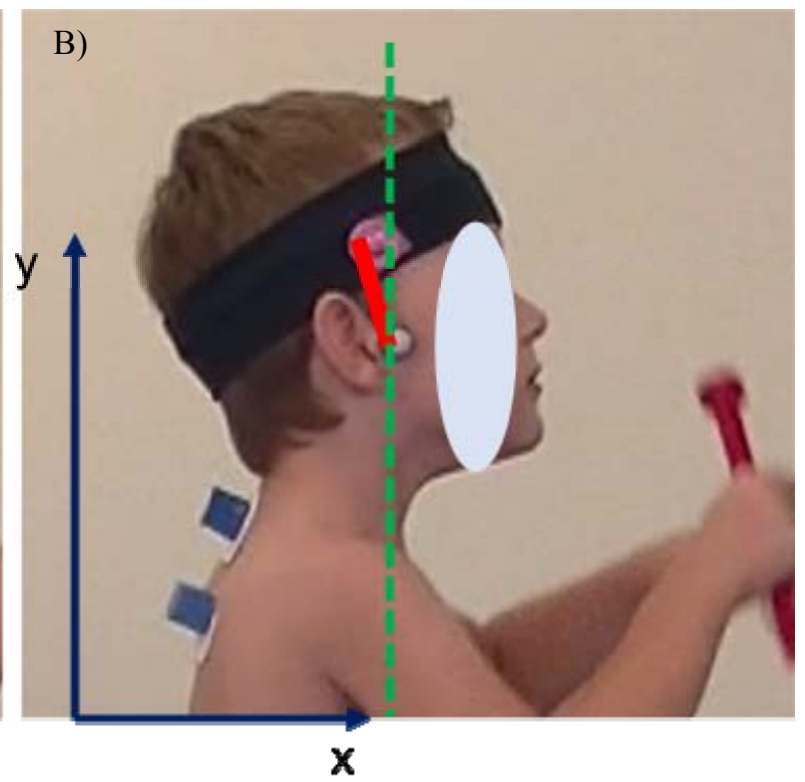

Fig. 1. Head segment and angle definition. Showing the head segment (bold red) and the real vertical (dashed green). A) Shows an aligned position of the head; B) shows head movement. 


\section{RESULTS}

Head motion during testing of Upper-Thoracic (UT) and Lower-Lumbar (LL) segmental levels are shown in Fig. 2

Head motion for the UT tests had larger values for the reactive test $\left(17.7^{\circ \mathrm{s}-1}, 14.1^{\mathrm{o} \cdot \mathrm{s}-1}\right.$ and $\left.26.4^{\circ \cdot \mathrm{s}-1}\right)$ of the three participants, than for the static $\left(6.2^{\circ \cdot s-1}, 4.7^{\circ \cdot s-1}\right.$ and $\left.4.6^{\circ \cdot s-1}\right)$ and active $\left(7.3^{\circ \cdot \mathrm{s}-1}, 8.3^{\circ \cdot \mathrm{s}-1}\right.$ and $\left.13.2^{\circ \cdot \mathrm{s}-1}\right)$ tests. At the LL segmental level, the poorer lumbar control of Child 2 and Child 3 was demonstrated in both active $\left(39.9^{\circ \cdot \mathrm{s}-1}, 23.3^{\circ \cdot \mathrm{s}-1}\right)$ and reactive tests $\left(37.3^{\circ \cdot s-1}, 13.8^{\circ \cdot s-1}\right)$.

\section{DISCUSSION}

The SATCo is a tool to systematically assess motor control of the trunk in the presence of a neuromotor disability. This study measured head motion during a SATCo to determine if measurement reflected clinical observations showing that head motion increased with external support at a lower trunk segment if the child did not have control at that segmental level. The video-based method presented confirmed that the coordinates from landmarks defining body segments (head markers) enabled generation of an objective measure that related to the clinical SATCo evaluation of control.

Child 1 was clinically determined to be learning full trunk control; the head motion values for both the UT and the LL segmental levels were similar. This corresponds to the clinical judgement that control was present at both segmental levels. Child 2 and Child 3 were clinically determined as learning control at the Upper-Lumbar segmental level having already acquired UT control. The head motion values, as with Child 1, reflect this clinical judgment. At the LL level, however, there are differences. The head motion values for the active and reactive trials are much larger for Child 2 and Child 3 than for Child 1 and corroborate the clinical judgement that greater head motion is seen if control is not present at a given segmental level. The measured values of head motion in Child 2 and Child 3 for the static trials at the LL segmental level, although greater than Child 1, were smaller than the active and reactive measures for these children. This may reflect early stages of control learning at this segmental level, adjacent to the subjectively assessed Upper-Lumbar segment. If so, this measurement of head motion could provide greater information than the subjective test alone.

The results of this pilot study also showed how increasing task complexity (static to reactive) and reducing the level of support (UT to LL), increases head motion in the presence of a neuromotor disability (Fig. 2). Lowering the level of support increases the number of trunk segments that must be under assured voluntary control to maintain an aligned sitting posture with the hands and arms free of external support.

Previous studies have quantified head motion of typically developing (TD) children in relation to the level of external support during quiet sitting $[10,11]$. Curtis et al showed mean sway values of $2.3^{\circ \cdot s-1}$ during unsupported sitting [10]; these values are smaller than the present head motion values for the static trials, but the children who participated in Curtis's study were TD children with assured sitting ability, while the children in the present study had cerebral palsy and were still learning to sit independently. Rachwani et al showed that the mean head motion was of $18^{\circ}$ for thoracic support and $30^{\circ}$ for pelvic support in TD children who were learning to sit independently [11]; these values are consistent with the head motion values found in this study, confirming that a lower external trunk support results in larger values of head motion when the child has not acquired full trunk control in sitting. Bigongiari et al described how the electromyographic (EMG) activity increased

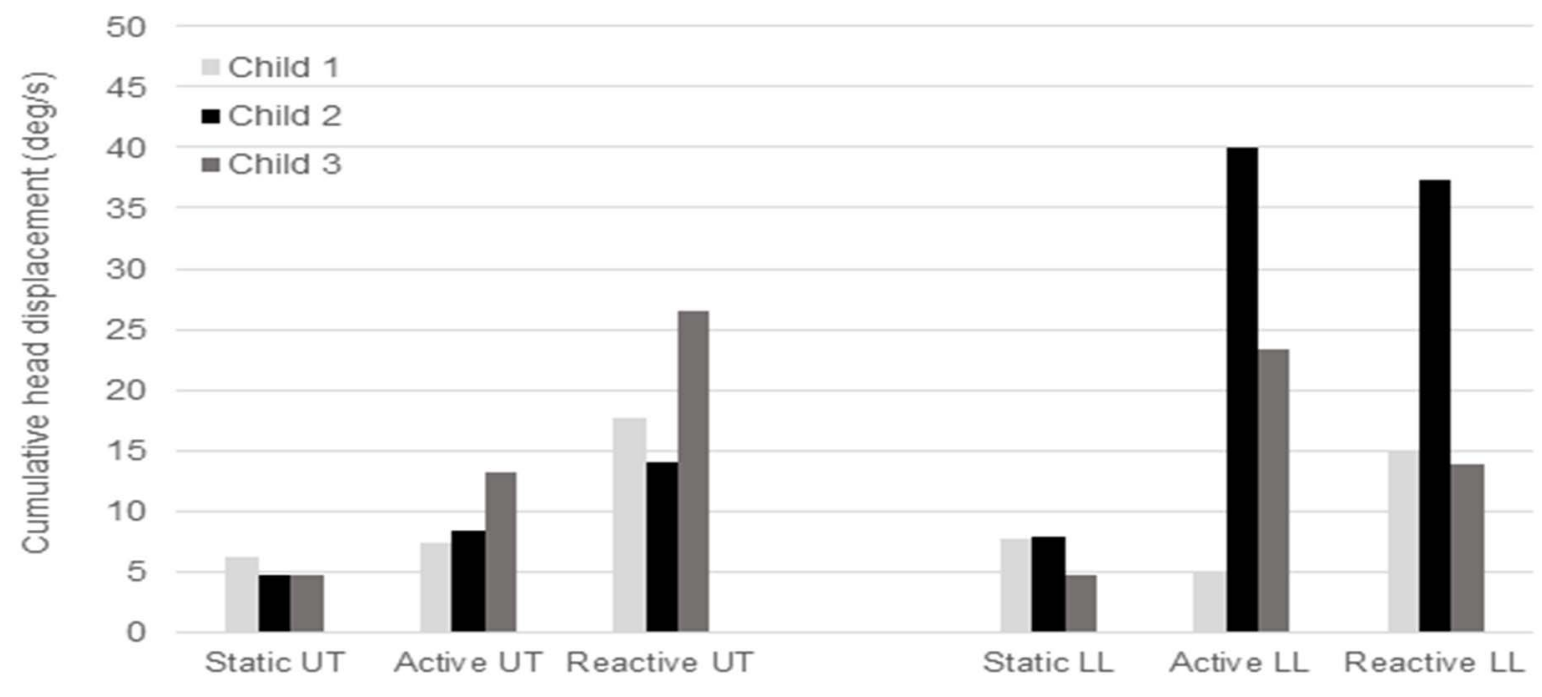

TRIAL TESTED

Fig. 2. Cumulative head motion. Showing the cumulative absolute displacement from the vertical normalised by time (deg $\cdot \mathrm{s}-1)$ of each child, for the UpperThoracic (UT) and Lower-Lumbar (LL) segmental levels, for the static, active and reactive components of control. 
from anticipatory to compensatory task in a group on children with CP [12]; the larger values of head motion in relation to the task complexity in this study are consistent with Bigongiari's findings. However, it is noted that these reported studies used a 3D motion capture system [10], magnetic tracking [11], and EMG [12], all of which are difficult and costly in a clinical setting with children with severe disability.

The measurement of head motion in this pilot study corroborated the clinical observation, suggesting that it could form an initial step towards the generation of an objective quantification of a subjective assessment. An objective clinicalfriendly tool should be easy to use for the therapists with minimal disruption to the normal clinical practice routine. A video-based system was used in the present study as it has the advantage of presenting an outcome that is similar to the human observation of posture; and because video recordings are already commonly used in clinical practice. In the present study, markers were required to identify a head segment and enable the calculation of head segmental angles. Although a fully automated objective tool should be markerless to avoid the limitations of missing markers when tracking on 2D videos [13], the results of the present study give confidence for future work towards a markerless system. The further development of a tool based on these principles, has the potential to complement clinical assessments currently used (in addition to SATCo) to support the therapeutic interventions in physiotherapy that will contribute to the evidence-based practice decision making.

\section{CONCLUSION}

This pilot study measured head motion during a SATCo, corroborating the subjective clinical assessment. The head motion calculations presented in this study are based on a simplified representation of the head. The present results show how the quantification of the angular movement of the head in relation to a real vertical, relate positively to the clinical assessment of control. The results also show how head motion relates to an increase of task complexity (static to reactive). This video-based approach represents a step towards the development of an objective tool for the assessment of head/trunk control in children with CP. Such objective tool development could have a major positive impact in the assessment of patients with neuromotor disabilities and in evaluating clinical physiotherapeutic interventions.

\section{ACKNOWLEDGMENT}

The authors express sincere thanks to the children and families who participated in this study and to the staff of The Movement Centre, Oswestry, UK.

\section{REFERENCES}

[1] Dawes M, Summerskill W, Glasziou P, Cartabellotta A, Martin J, Hopayian K, et al. Sicily statement on evidence-based practice. BMC Med Educ. 2005;5:1.

[2] Shumway-Cook A, Woollacott MH. Constraints on motor control: an overview of neurologic impairments. In: Shumway-Cook A, Woollacott $\mathrm{MH}$, editors. Motor control: translating research into clinical practice. 4th ed. Philadelphia, Pa: Lippincott Williams \& Wilkins; 2011. p. 104-40.

[3] Curtis DJ, Butler P, Saavedra SL, Bencke J, Kallemose T, Sonne-Holm $\mathrm{S}$, et al. The central role of trunk control in the gross motor function of children with cerebral palsy: A retrospective cross-sectional study. Dev Med Child Neurol. 2014;57:351-7.

[4] Jensen J, van Zandwijk R. Biomechanical aspects of the development of postural control. In: Korff $\mathrm{T}$, De Ste Croix M, editors. Paediatric biomechanics and motor control: theory and application. Abingdon, Oxon: Routledge research in sport and exercise science; 2012. p. 139-59.

[5] Pountney TE, Cheek L, Green E, Mulcahy C, Nelham R. Content and Criterion Validation of the Chailey Levels of Ability. Physiotherapy. 1999;85:410-6.

[6] Russell DJ, Rosenbaum PL, Avery LM, Lane M. Gross motor function measure (GMFM-66 and GMFM-88) user's manual. First ed. London: Mac Keith Press; 2002.

[7] Heyrman L, Molenaers G, Desloovere K, Verheyden G, De Cat J, Monbaliu E, et al. A clinical tool to measure trunk control in children with cerebral palsy: the Trunk Control Measurement Scale. Res Dev Disabil. 2011;32:2624-35.

[8] Reid DT. Sitting Assessment for Children with Neuromotor Dysfunction SACND. San Antonio, Texas: Therapy Skill Builders; 1997.

[9] Butler P, Saavedra SL, Sofranc M, Jarvis S, Woollacott MH. Refinement, reliability, and validity of the Segmental Assessment of Trunk Control. Pediatr Phys Ther. 2010;22:246-57.

[10] Curtis DJ, Hansen L, Luun M, Loberg R, Woollacott MH, Saavedra SL, et al. Measuring postural sway in sitting: a new segmental approach. J Mot Behav. 2015;47:427-35.

[11] Rachwani J, Santamaria V, Saavedra SL, Wood S, Porter F, Woollacott MH. Segmental trunk control acquisition and reaching in typically developing infants. Exp Brain Res. 2013;228:131-9.

[12] Bigongiari A, De Andrade e Souza F, Franciulli P, El Razi Neto S, Correa R, Mochizuki L. Anticipatory and compensatory postural adjustments in sitting in children with cerebral palsy Hum Mov Sci. 2011;30:648-57.

[13] Sánchez MB, Loram I, Darby J, Holmes P, Butler PB. A video based method to quantify posture of the head and trunk in sitting. Gait Posture. 2017;51:181-7. 